\title{
Perceptions of Community Health Workers (CHW) On Barriers And Enablers To Care For People With Psychosis In Rural Mozambique: Findings of a Focus Group Discussion Study Using The Capability, Opportunity, Motivation and Behavior Framework (COM-B Framework).
}

Dirceu Mabunda ( $\nabla$ dihepama@gmail.com )

Federal University of São Paulo

Déborah Oliveira

Inter-American Development Bank

Mohsin Sidat

Eduardo Mondlane University

Francine Cournos

Columbia University

Milton Wainberg

Columbia University

Jair de Jesus Mari

Federal University of São Paulo

Research Article

Keywords: Community health workers, psychosis, enablers, barriers, capacity, opportunity, motivation, behavior

Posted Date: December 15th, 2021

DOI: https://doi.org/10.21203/rs.3.rs-1163377/v1

License: (1) This work is licensed under a Creative Commons Attribution 4.0 International License. Read Full License 


\section{Abstract}

Background: Psychotic disorders contribute significantly to the global disease burden by causing disability, impaired quality of life, and higher mortality in affected people compared with the general population. In rural settings, where there is limited or no access to healthcare, individuals living with psychotic disorders often seek support from Community Health Workers (CHWs). However, little is known about what CHWs know about psychosis and how they manage such cases. This study aimed to explore the CHWs perception of psychosis and their experiences and beliefs about the factors that might enable or hinder care taking for patients with psychosis in rural settings in Mozambique.

Methods: A qualitative study was conducted in rural districts of Maputo Province, a southern region of Mozambique, using six focus group discussions with participation of $79 \mathrm{CHWs}$. Thematic analysis was used informed by the Capabilities, Opportunities, Motivation and Behavior framework (COM-B).

Results: Nine primary themes were identified. Overall, CHWs perceived psychosis as treatable medical conditions and held a positive attitude about being part of the care taking process of patients with psychosis in rural settings. Partnerships with key-stakeholders such as traditional healers, health care workers and families, were perceived by $\mathrm{CHWs}$ as enablers to improve access to care in rural areas. However, stigma, myths, and lack of competencies to treat people with psychosis were perceived by CHWs as barriers for appropriate care.

Conclusion: $\mathrm{CHWs}$, with adequate support, can play an important role in the care of patients with psychosis in rural settings, including identification of patients requiring care and referring them to appropriate healthcare professionals, and follow-up of medicated patients with psychosis within the rural remote setting. Training of CHWs should consider inclusion of basic mental health care competencies.

\section{Introduction}

Psychotic disorders contribute significantly to the global disease burden by causing disability, impaired quality of life, and higher mortality in affected people compared with the general population (1)'(2). Schizophrenia is the main cause of psychosis and has been identified as a priority disorder by the Global Mental Health Initiative (3). Nevertheless, the median treatment gap for schizophrenia in low and middleincome countries is approximately $70 \%$ (4) and a scarcity of human resources for the treatment of psychosis has been identified as a contributor to this existing gap globally (5).

Mozambique is a low-income country where about two-thirds of its total population (32 million people in 2020) live in rural areas. In Mozambique, the prevalence of psychosis is regarded as being higher in rural compared to urban areas (6). Schizophrenia is the leading cause of inpatient admission to psychiatric hospitals and the second most common reason for psychiatric outpatient consultation (7). Family support, community-based rehabilitation, self-help and support groups are some of the key priority interventions to address the burden of mental, neurological and substance use disorders in people living with psychosis (8). Interventions delivered by Community Health Workers (CHW) can effectively reduce 
disability and increase adherence to treatment in people living with schizophrenia (9). The effectiveness and feasibility of community-based interventions for people living with psychosis have been demonstrated in Latin America (10), Asia (11) and Africa (12).

The CHW program (Programa dos Agentes Polivalentes Elementares-APEs) in Mozambique was created in 1978, informed by the primary health care approach, with the aim of expanding access to health care in remote and rural regions (13). After a challenging period followed by a civil war in the country (19771992), the program was relaunched in 2010 with a defined training package, supervision strategies and logistical support (14). However, the mental health and neurological component is still inadequately addressed, with only a brief reference to epilepsy (15). Many people with psychosis will start treatment elsewhere and then return to their communities requiring continued attention and follow up. In rural areas, this attention is scarce or non-existent. We focused on $\mathrm{CHWs}$ because they are important health care providers in rural areas of Mozambique (16). Having CHWs as providers of mental health interventions for people living with psychosis in rural areas could potentially contribute to narrowing the gap in mental health care in Mozambique. However, studies about the knowledge and perceptions of CHWs on the barriers and enablers of mental health care for people living with psychosis in Mozambique are scarce.

\section{Aim}

The study aimed to explore the CHWs perception of psychosis and their experiences and beliefs about the factors that might enable or hinder care taking for patients with psychosis in rural settings in Mozambique.

\section{Theoretical framework}

The model of behaviour change "Capability, Opportunity, Motivation and Behavior" (COM-B) (17) was used to inform our focus group guide and data analysis. Our goal was to better understand the interactions between capability, opportunity and motivation on behaviour that could enable access to health care. COM-B can be used to facilitate the design of interventions (17) aimed at improving access to healthcare by people living with psychosis in rural settings of Mozambique. In this framework, capability includes the necessary knowledge and skills that enable a person's psychological and physical capacity to engage in an activity. Motivation includes the brain processes that enable direct behaviour, including habitual processes, emotional responses and analytical decision making. Opportunity encompasses all the external factors which make the behaviour possible.

\section{Methods}

\section{Study design}

A qualitative study was carried out and data collected through focus group discussions with $\mathrm{CHWs}$. The focus groups were regarded as appropriate as they allowed for the probing of specific issues in group interaction and clarification of individual and shared perspectives of health issues (18). This sharing of 
experiences enhances additional thoughts and insights between group discussion participants (CHWs), providing opportunities to make connections and learn from shared experiences during the group dynamics.

\section{Sample and recruitment}

Using a purposive sampling strategy (19), we identified and subsequently invited all CHWs from the three districts to participate voluntarily. Those that agreed were additionally informed about the time and place where the focus group discussions would occur. Recruitment was led by DM and took place during monthly district meetings at district health clinics between September and December 2019. Travel expenses were reimbursed, but no other incentives were offered. The objectives of the study were explained to the CHWs who agreed to participate, and informed consent was obtained. Discussions were facilitated in the Portuguese language by DM, a male researcher trained in qualitative methods with no previous contact with the participants. Each session was audio-recorded and transcribed verbatim by DM. In addition to these recordings, written notes were also taken, to allow for future review during data analysis and other research processes and to ensure that participants views were clearly documented.

\section{Procedure}

The study was approved by the National Review Board (92/CNBS/2017) and all participants signed the informed consent prior to the focus group discussion. The interview guide was designed by the research team and informed by the COM-B framework. Before the study commenced, the focus group guide was piloted in other districts which were not part of the study, so that it could be refined and revised. Because CHWs are not trained in mental health themes, we adapted the mhGAP case vignette of psychosis (20) to provide a unified description of the common experiences of psychosis. Participants were asked to identify if they have had previous experiences with similar cases and identify the causes (Supplementary material).

\section{Study setting}

Three rural districts of Maputo province in southern region of the country were included: Manhiça, Moamba and Boane.

Manhiça district is located in the north area of Maputo Province, $80 \mathrm{~km}$ from Maputo City. It has about 214,751 inhabitants. It has 15 primary care units. Moamba district is located to the north area of Maputo Province, $52 \mathrm{~km}$ from Maputo City. It has about 56,559 inhabitants. It has 11 primary care units. Boane district is located in the southeastern region of Maputo Province, $30 \mathrm{~km}$ from Maputo City. It has about 134,006 inhabitants. It has 10 primary health care units.

\section{Data analysis}

Transcripts were analysed manually using a word template based on guidelines for the thematic analysis(21). The following steps were taken to analyse the data: 1) familiarization: the first author (DM) 
revisited the focus group discussion audio and verbatim transcript several times to familiarize himself with the data; 2) code generation: the coding was derived from the data using the structural coding approach (22);3) theme search: after initial coding, a set of overarching themes were developed; 4) theme revision: revision of the coding process was performed, during which themes were refined and new themes could be added; 5) theme definition and naming: consultation with a second researcher (DO) was undertaken to refine the way each theme was developed; and 6) thematic final map: this map was used to inform the reporting of the analysis. Data saturation was assessed following Monike Hennik's guidelines on saturation (23) which states that a study where focus group are not stratified by any characteristic will require a small sample size to reach saturation (3-6 groups). Reporting followed the Consolidated Criteria for Reporting Qualitative Research (18).

\section{Results}

Seventy-nine representative $\mathrm{CHWs}$ took part in six focus groups (group size $n=5-13$ ), and each focus group lasted between 65 to 100 minutes. The socio-demographic characteristics of the sample are described on Table 1.

Table 1. Socio demographic characteristics of participants 


\begin{tabular}{|ll|}
\hline Variable & Focus group $(\mathrm{n}=79)$ Mean (SD) \\
\hline Age (years) & $38.81(11.92)$ \\
\hline Time as CHW (years) & $7.71(6.91)$ \\
\hline Gender $\mathrm{n}(\%)$ & \\
\hline Male & $28(35.4)$ \\
\hline Female & $51(64.6)$ \\
\hline District & \\
\hline Boane & $13(16.5)$ \\
\hline Manhiça & $34(43)$ \\
\hline Moamba & $32(40.5)$ \\
\hline Qualification & \\
\hline Primary & $36(45.6)$ \\
\hline Basic & $26(32.9)$ \\
\hline Midlevel & $7(8.9)$ \\
\hline No information & $10(12.7)$ \\
\hline Marital status $\mathrm{n}(\%)$ & \\
\hline Single & $53(67)$ \\
\hline Married & $24(30.5)$ \\
\hline Divorced & $2(2.5)$ \\
\hline
\end{tabular}

Abbreviations: CHW- Community Health Workers; SD-Standard deviation; n-number;

$\%$ - percentage.

Nine primary themes were identified: knowledge about psychosis and mental health; the manifestations of the disease; attitudes and practices towards patients with psychotic disorders; key-stakeholders in the care of patients with psychotic disorders; perceived needs for training; perceived readiness to provide care for patients with psychosis; community perceptions; and beliefs and attitudes related to psychosis. Table 2 shows details of COM-B model related themes.

Table 2: COM-B model and related themes 


\section{COM-B \\ components \\ (Michie et \\ al., 2011)}

COM-B

components

applied to CHW

views of

enablers and

barriers

Themes from this Illustrative quotes study

\section{CHW knowledge \\ towards \\ psychosis \\ Knowledge about the cause of psychosis:}

The nature of the illness

Alcohol and Substance Abuse as a cause of psychosis

Post Malaria
Infection in
Childhood

Familiar heritage and young age; Familiar problems

Trauma (sexual trauma, stress, anger)

Bad spirits,
punishment
Isolation,
discrimination,
orphaned in
childhood

The nature of the disease: the lack of insight that characterize many people with psychosis. It includes agitation, delusions, hallucination and bizarre behavior.
"To my mind, such illness strikes young people [the most], since most young people use suruma /cannabis sativa a lot, so many are there due to their using cannabis sativa." FGD1, 01

"To my mind, these problems are rooted in the families, perhaps. Suppose, perhaps, there are families who have had cases of that illness, and maybe they think that such persons would pass it on to others." FG 207

"In most-people's minds, this is witchcraft, in other words, someone has been bewitched...the community thinks that this illness is rooted in tradition." FG 203

"I can't tell, and scientifically speaking, it is difficult to tell. Still, it seems that a person, in particular kids, for instance, who had cerebral malaria earlier, right, can develop this illness, scientifically speaking... I cannot tell whether or not myths become a reality, but what people have said is that these illnesses have to do with evil spirits." FGD 5, 03.

"Consider the example of someone who is discriminated where they live, they may eventually suffer from 
this illness. If he/she feels lonely without being hugged by their relatives, he/she may develop this illness." FGD 206

"Someone with mental disorders can, for instance, serve food on a plate, then dump it onto the floor, collect the food from the floor and eat it. All that is normal is seen by them as something abnormal, as I explained earlier." FGD 056

Physical capability: physical

skills to enact the behavior
Healthcare professionals' skills to support people with psychosis and their family's inability to access the clinic due to lack of income
Training need: need to train the APEs to diagnose and manage psychosis. Include training in screening, brief intervention and referral of the patients with psychosis as well as follow up.
Opportunity:

'the factors

that lie outside of the individual that make behavior possible or prompt it'.
Social

opportunity: 'afforded by the cultural milieu' which 'dictates the way we think about things

\section{Social}

barriers/enablers to $\mathrm{CHW}$ care for people with psychosis

\section{Attitude and} practice of CHWs: the attitude of APEs towards psychosis. Views of CHWs towards people with psychosis. Positive attitude of the CHWs towards people with psychosis.

Partnership: CHWs, traditional healers, family and community working together.

\section{Engage families in care pathway (enabler)}

"We are not yet well prepared on the topic, right? Given that we are not knowledgeable about the topic, we shall be prepared, well prepared after undergoing a small training." FGD 068
"But we as CHWs have been entrusted by the community with working for them. We already have several types of packages with which we are working. We're going to give the case a try until we succeed, because that person belongs to the community, that is a person of ours." FGD 1 04

"Because when this sort of illness appears, they think that this illness can't be cured at the hospital, but that the illness can only be cured by traditional healer(s). We are not refusing this. We are saying yes, you can go 
and see traditional

healers, don't stop seeing them, but first go to the hospital."

FGD 016

"It's not easy for the community to understand that this illness can be treated at the hospital, but whenever a visit is paid by us we should give advice, give support so that they take him/her to the hospital, to see whether they can eventually understand it. $\mathrm{He} / \mathrm{sh}$ will end up accepting the pieces of advice and whenever he/she is taken to hospital he/she will see that things will change." FGD 052

"As for my community, I, as a CHW, have to help those people who do not understand that the illness can be treated at the hospital, advise the ladies or the families to take the person with that disability to hospital, in order to get better..." FGD 028

"We have to make more auxiliary visits to help our communities. The communities don't know where else that illness comes from... As CHWs we have to help the community by raising their awareness so that community members can also know that a person can get ill and not be in a spell, but that at the hospital he/she can be cured." FG2 02

"Yes, because the difficulties are the fact that communities already share the 
conviction that this kind of illness is not treated at a hospital. That is the most common difficulty, all the more so because they tell us that if they go there, they are going to be sent to Magude, and from Magude, they will be sent to Maputo. Thus, they don't have money to go there."

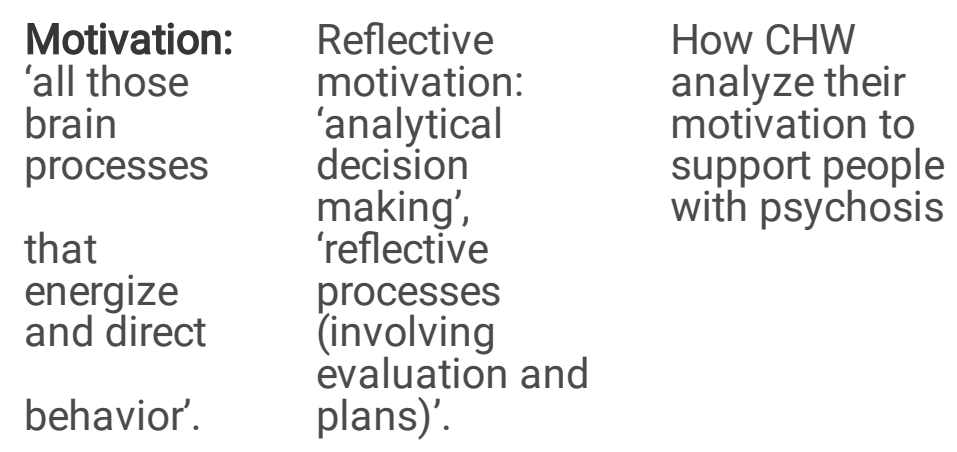

\section{Lack of confidence (barrier)}

Readiness of CHW
to provide mental
health care
(enabler): Positive attitude of $\mathrm{CHW}$ towards psychosis

Readiness to provide care for people with psychosis
"As we do not know enough about the illness, if we approach a family to offer explanations, family members in the first place would want to know what is happening. These are questions which, at that moment, we would not be able to answer. And our credibility as CHWs there is virtually nil." FGD 06 12".

"Again, we CHWs are not known there in the community as persons dealing with these stuff, except for dealing with malaria, cholera, diarrhoea. Meanwhile, nowadays, if we approach them with this topic, they will ask us where we got this information from, and that we are lying."

"But we as CHWs have been entrusted by the community with working for them. We guarantee that we will do what we have been doing with those other packages, because so far we have already been achieving the goals. We're not going to set someone aside in that it's difficult. We're going to give the case a try until we succeed, because that 
person belongs to the community, that is a person of ours." FGD 1 04

Automatic
motivation:
'involving
emotions and
impulses that
arise from
associative
learning and/or
innate
dispositions.

How

professionals

are motivated to

support health

behaviors in

relation to their

own (perhaps

unconscious)

emotions or

understandings

about those

behaviors

\section{Stigma issues (barriers)}

"I felt very sorry when two months ago a boy who is still old enough to go to school or do what is for his own good, received an unsatisfactory response from his father towards his illness, as the father tied him up and beat him up a lot and put him on his minibus." FGD 104

"As far as recovery is concerned, the difficulty lies with the family itself, because when the family sees the kid in the state he/she is messing up everything, they think he/she only does that because he/she wants to [do so], but no, that is an illness." FGD 104

"The communities don't know where else that illness comes from. People say that thing that somebody got bewitched. The thing is that there are people who say that cure can't be found there, you can only go to a traditional healer and be given I don't know what." FG2 02

"Another thing involves the community: It is the fact that we were born there, grew up there, are known by people, people know what we do in our homes, people are aware that we are poor. So, when we approach the community, they don't take us seriously-they think that we are lying; 
They despise us." FGD 0210

"Another thing involves the community: It is the fact that we were born there, grew up there, are known by people, people know what we do in our homes, people are aware that we are poor. So, when we approach the community, they don't take us seriously-they think that we are lying; I'm not sure, as they know us (...). They despise us." FGD 0210

\section{Capability}

Capability can be explained as the CHW's capacity to engage in the management of mental health issues for people with psychosis in rural settings. Two barriers and one facilitator emerged in relation to this construct. Three themes were identified within the category of capability: knowledge about the cause of psychosis, the nature of the disease, and lack of mental health skills.

\section{Knowledge about cause of psychosis}

To determine levels of knowledge about psychosis, participants were asked to define the causes of psychosis. Generally, CHWs demonstrated limited knowledge and understanding of psychosis. Most $\mathrm{CHWs}$ indicated that alcohol use and substance abuse can be important causes of psychosis or worsening of symptoms. The theme related to knowledge about the causes of psychosis encompassed 5 sub-themes: alcohol and substance abuse; malaria infection in childhood; family inheritance; young age; and family problems, such as trauma (sexual trauma, stress, anger), bad spirits, punishment, and isolation (discrimination, orphaned in childhood).

The main substance implicated in the development of symptoms was cannabis sativa "suruma":

"To my mind, such illness strikes young people [the most], since most young people use suruma /cannabis sativa a lot, so many are there due to their using cannabis sativa." FGD1, 01

CHWs also observed that psychosis is caused by heritability from fathers to children, besides the use of drugs.

"To my mind, these problems are rooted in the families, perhaps. Suppose, perhaps, there are families who have had cases of that illness, and maybe they think that such persons would pass it on to others." FG 207 
Few CHWs believed that psychosis is caused by witchcraft and possession by bad spirits:

"In most-people's minds, this is witchcraft, in other words, someone has been bewitched...the community thinks that this illness is rooted in tradition." FG 203

One $\mathrm{CHW}$ observed that psychosis could be the result of malaria infection during the childhood, however, did not discard the possibility of psychosis being the result of "evil spirits":

"I can't tell, and scientifically speaking, it is difficult to tell. Still, it seems that a person, in particular kids, for instance, who had cerebral malaria earlier, right, can develop this illness, scientifically speaking... I cannot tell whether or not myths become a reality, but what people have said is that these illnesses have to do with evil spirits." FGD 5, 03.

Discrimination and lack of empathy was stated as a cause of psychosis by one CHW:

"Consider the example of someone who is discriminated where they live, they may eventually suffer from this illness. If he/she feels lonely without being hugged by their relatives, he/she may develop this illness." FGD 206

\section{Training needs}

CHWs felt that they lacked the skills needed to care for people with psychosis and suggested practical and theoretical training to overcome this gap:

"We are not yet well prepared on the topic, right? Given that we are not knowledgeable about the topic, we shall be prepared, well prepared after undergoing a small training." FGD 068

\section{The nature of the disease}

Participants considered that the ability to provide care for people with psychosis could be influenced by the nature of the disease. One CHW recognized that it is difficult to deal with people with psychosis because of their symptoms and behaviour:

"Someone with mental disorders can, for instance, serve food on a plate, then dump it onto the floor, collect the food from the floor and eat it. All that is normal is seen by them as something abnormal, as I explained earlier." FGD 056

\section{Opportunity:}

Three enablers emerged as influencing CHWs' opportunities within this construct: attitudes and practices of CHWs towards psychosis; partnerships; and engaging families in the care pathway.

\section{Attitude and practices of $\mathrm{CHW}$}

CHWs felt that are empowered and motivated to care for people living with psychosis: 
"But we as CHWs have been entrusted by the community with working for them. We already have several types of packages with which we are working. We're going to give the case a try until we succeed, because that person belongs to the community, that is a person of ours." FGD 104

\section{Partnerships}

Partnering with families, traditional healers and health care workers was a way that $\mathrm{CHWs}$ reported to improve access and care for people living with psychosis in their communities:

"Because when this sort of illness appears, they think that this illness can't be cured at the hospital, but that the illness can only be cured by traditional healer(s). We are not refusing this. We are saying yes, you can go and see traditional healers, don't stop seeing them, but first go to the hospital." FGD 016

\section{Engage families in care pathway}

Almost all $\mathrm{CHW}$ recognize that they have a challenge in convincing families to engage in the care of their kids with psychosis. They also recognize that families need support from the community and all stakeholders to fulfil the needs of people living with psychosis:

"It's not easy for the community to understand that this illness can be treated at the hospital, but whenever a visit is paid by us we should give advice, give support so that they take him/her to the hospital, to see whether they can eventually understand it. He/she will end up accepting the pieces of advice and whenever he/she is taken to hospital he/she will see that things will change." FGD 052

Education and providing support for people with psychosis and their caregivers were seen by CHWs as enablers for recovery:

"As for my community, I, as a CHW, have to help those people who do not understand that the illness can be treated at the hospital, advise the ladies or the families to take the person with that disability to hospital, in order to get better..." FGD 028

CHWs are able to support the communities to better understand the causes of psychosis and increase awareness about mental health issues:

"We have to make more auxiliary visits to help our communities. The communities don't know where else that illness comes from... As CHWs we have to help the community by raising their awareness so that community members can also know that a person can get ill and not be in a spell, but that at the hospital he/she can be cured." FG2 02

CHWs stated that families who care for people with psychosis are reluctant to go to clinic because they are not comfortable with continuing through the complex referral and treatment pathway:

"Yes, because the difficulties are the fact that communities already share the conviction that this kind of illness is not treated at a hospital. That is the most common difficulty, all the more so because they tell us 
that if they go there, they are going to be sent to Magude, and from Magude, they will be sent to Maputo. Thus, they don't have money to go there." FGD 028.

\section{Motivation.}

Two barriers and one facilitator were categorized as influencing participants' motivation to manage people with psychosis.

The motivations that were expressed by $\mathrm{CHWs}$ to improve care for people living with psychosis were positive and enabling regarding the readiness of $\mathrm{CHWs}$ to provide mental health care for people with psychosis.

\section{Lack of confidence}

CHWs perceived a lack of confidence and skills/ability to care for people with psychosis and their families as barriers. This reduces the self-efficacy of $\mathrm{CHWs}$ to care for people with psychosis. Building that capacity and professional competence were seen as important factors for $\mathrm{CHW}$ motivation:

"As we do not know enough about the illness, if we approach a family to offer explanations, family members in the first place would want to know what is happening. These are questions which, at that moment, we would not be able to answer. And our credibility as CHWs there is virtually nil." FGD 0612 ".

Because CHWs so far have not address mental illness in the community, CHWs felt that households where a family member is a person living with psychosis would not trust them as a source of knowledge:

"Again, we CHWs are not known there in the community as persons dealing with these stuff, except for dealing with malaria, cholera, diarrhoea. Meanwhile, nowadays, if we approach them with this topic, they will ask us where we got this information from, and that we are lying."

\section{Readiness of CHW to provide mental health care}

CHWs perceived that they would be able to raise awareness within the community and among families of people with psychosis in a way that increases knowledge about psychosis. Additionally, they felt that they were able to care for patients with psychosis and help their communities:

"But we as CHWs have been entrusted by the community with working for them. We guarantee that we will do what we have been doing with those other packages, because so far we have already been achieving the goals. We're not going to set someone aside in that it's difficult. We're going to give the case a try until we succeed, because that person belongs to the community, that is a person of ours." FGD 104

\section{Stigma issues}

Stigma issues were recognized by $\mathrm{CHWs}$ as barriers for access, diagnosis, recovery and continuity of care by people living with psychosis. CHWs felt that some families of people living with psychosis treat them 
with dehumanization and blame:

"I felt very sorry when two months ago a boy who is still old enough to go to school or do what is for his own good, received an unsatisfactory response from his father towards his illness, as the father tied him up and beat him up a lot and put him on his minibus." FGD 104

Families in the rural communities may express their stigma about psychosis by sanctioning violent acts against people manifesting psychotic symptoms. One CHW stated that people don't believe that mental disorders exist. They think that it's a personal choice to have a mental illness:

"As far as recovery is concerned, the difficulty lies with the family itself, because when the family sees the kid in the state he/she is messing up everything, they think he/she only does that because he/she wants to [do so], but no, that is an illness." FGD 104

The CHWs also recognized that misinterpretation about the origin/ causes of the psychosis may negatively impact recovery and create barriers to accessing mental health services and care:

"The communities don't know where else that illness comes from. People say that thing that somebody got bewitched. The thing is that there are people who say that cure can't be found there, you can only go to a traditional healer and be given I don't know what." FG2 02

CHWs felt discriminated against by some community members because they are members of the same community and mental health themes are not part of their current intervention package:

"Another thing involves the community: It is the fact that we were born there, grew up there, are known by people, people know what we do in our homes, people are aware that we are poor. So, when we approach the community, they don't take us seriously-they think that we are lying; They despise us." FGD 0210

\section{Discussion}

This qualitative study aimed to explore the CHWs perception of psychosis and their experiences and beliefs about the factors that might enable or hinder care taking for patients with psychosis in rural settings in Mozambique. It sheds light on how CHWs understand psychotic illnesses, their desires for further education and skills to improve their understanding and how they approach psychotic illnesses, and how a lack of knowledge and misunderstandings about the causes of psychosis in these rural communities hampers appropriate approaches to deal with stigma and prevent abuse of people with psychosis.

Our findings suggest that $\mathrm{CHWs}$ have a good attitude and perceive themselves as being ready to provide mental health care for people with psychosis and that they are available to be trained to perform this additional task. Furthermore, CHWs also consider that having a good collaboration between $\mathrm{CHWs}$, families, traditional healers and health care professionals is an important enabler to engage/maintain people with psychosis with appropriate care while residing in remote rural setting. 
The study shows that CHWs with appropriate knowledge about psychosis had better attitudes and practices towards psychosis. Many CHWs were aware of factors that may contribute to the onset of psychotic illnesses, such as the abuse of alcohol and illicit substances, especially marijuana, the role of cerebral malaria in contributing to mental illness, and the presence of other contributing factors, such as trauma. CHWs were able to discuss community beliefs about witchcraft and spirit possession as factors that needed to be addressed appropriately to promote better acceptance of psychiatric treatment in rural communities. These findings are in line with other studies (24).

A systematic review suggests that family and community support for people with psychosis results in good outcomes and recovery (25). In this study, family support has been stated as an important enabler to recovery of people with psychosis. This was also suggested in previous studies (27)'(28). CHWs in this study faced issues of lack of knowledge and skills to care for people with psychosis and suggested training in mental health themes. Our study is in line with other studies that were carried out in Kenya about the helpfulness of interventions that improve knowledge among $\mathrm{CHWs}$ through training on the diagnosis of psychosis (26).

A randomized controlled trial in Nigeria and Ghana suggested that collaborative care between traditional healers and primary health-care workers was more effective and cost-effective for care for people with psychosis in low resource settings (12).

The goal of our study was to explore the views of CHWs concerning the barriers and enablers of access to mental health services and recovery services for people with psychosis in their communities. Our findings shed important light on the negative impact of the stigma on access to mental health care and recovery in rural areas of Mozambique. They suggest the need for improved collaboration/partnerships between families, traditional healers, CHWs and health care workers. Discrimination related to psychosis was an important barrier that contributed to low levels of knowledge towards psychosis among CHWs (26).

Additionally, CHWs found it challenging to provide care for people with psychosis, but they also felt many barriers could be lowered by training $\mathrm{CHWs}$ on mental health themes and on techniques for engaging/ convincing families to be involved in the care pathway for people with psychosis. One previous study found that training for $\mathrm{CHWs}$, along with providing family support and community mobilization, had a positive impact on the quality of life of people with schizophrenia (29). Some CHWs felt discriminated against by their communities when providing mental health care, highlighting the impact of stigma on those who are caring for people with psychosis.

\section{Limitations}

The sample was composed by $\mathrm{CHWs}$ of the southern region of Mozambique and there are considerable ethno-cultural differences within different parts of the country which limits the applicability of findings to the whole of the country. These CHWs' suggestions and opinions may not reflect those of CHWs in other 
contexts elsewhere in Africa or globally. However, this study is the first to explore the barriers and enablers of mental health care for people with psychosis living in rural settings in Mozambique from the perspective of CHWs. This study can serve as a background for further research focusing on the feasibility of implementing evidence-based interventions for people with psychosis in rural areas of Mozambique. The participation of $\mathrm{CHWs}$ in care of patients with psychosis is of paramount importance as they are the ones who deliver basic health care and first point of contact with the health system in the rural areas of Mozambique.

\section{Conclusion}

In conclusion, this study presents exploratory evidence of the role that CHWs could play in participating in care of patients with psychosis. CHWs, with adequate support, can play an important role in the care of patients with psychosis in rural settings, including identification of patients requiring care and referring them to appropriate healthcare professionals, and follow-up of medicated patients with psychosis within the rural remote setting. Training of $\mathrm{CHWs}$ should consider inclusion of basic mental health care competencies.

\section{Abbreviations}

CHW: Community Health Workers

COM-B: Capability, Opportunity, Motivation and Behavior

APE- Agente Polivalente Elementar

CNBS- Comitê Nacional de Bioética em Saúde

FGD- Focus Group Discussions

\section{Declarations}

Acknowledgments: We would like to thank the Maputo Health Directorate for the support during the field work. We also would like to thank all Community Health Workers (APEs) who participate in the study.

Authors' Contributions: DM, MS, JM defined the aims of the study and wrote the protocol. All authors contributed to data interpretation, writing and editing. All authors approved the final version for submission.

Funding: This study is part of doctorate of DM. DM is D43TW009675 a NIMH - Fogarty grant for the "PALOP Mental Health Implementation Research Training" fellow.

Availability of Data and Materials: the data are available under reasonable request addressed to Dirceu Mabunda (dihepama@gmail.com). 


\section{Ethics approval and consent to participate}

All data collection protocols were approved by the National Review Board (92/CNBS/2017). The participants provided written informed consent for participation and recording prior to all data collection.

Consent for publication: Not Applicable

Competing interests: The authors report no conflicts of interest.

\section{References}

1. Stubbs B, Koyanagi A, Veronese N, Vancampfort D, Solmi M, Gaughran F. Physical multimorbidity and psychosis: comprehensive cross sectional analysis including 242, 952 people across 48 low- and middle-income countries. BMC Med. 2016;1-12.

2. Jongsma H, Kirkbride JB. Treated Incidence of Psychotic Disorders in the Multinational EU-GEI Study. JAMA Psychiatry. 2017;1-11.

3. Patel V. Universal Health Coverage for Schizophrenia: A Global Mental Health Priority. Schizophr Bull. 2016;42(4):885-90.

4. Lora A, Kohn R, Levav I, Mcbain R, Saxena S, Lora A. Service availability and utilization and treatment gap for schizophrenic disorders: a survey in 50 low- and middle-income countries. Bull World Health Organ. 2012;(October 2011):47-54.

5. Mari JDJ, Razzouk D, Thara R, Eaton J, Thornicroft G. Packages of care for schizophrenia in low- and middle-income countries. PLoS Med. 2009;6(10).

6. Patel V, Simbine APF, Soares IC, Weiss H a, Wheeler E. Prevalence of severe mental and neurological disorders in Mozambique: a population-based survey. Lancet [Internet]. 2007 Sep 22;370(9592):1055-60. Available from: http://www.ncbi.nlm.nih.gov/pubmed/17889244

7. Santos PF, Wainberg ML, Caldas de Almeida JM, Saraceno B, Mair J de J. Overview of the mental health system in Mozambique: addressing the treatment gap with a task-shifting strategy in primary care. Int J Ment Health Syst. 2016;1-9.

8. Patel V, Chisholm D, Parikh R, Charlson FJ, Degenhardt L, Dua T, et al. Addressing the burden of mental, neurological, and substance use disorders: Key messages from Disease Control Priorities, 3rd edition. Lancet. 2015;387(10028):1672-85.

9. Chatterjee S, Naik S, John S, Dabholkar H, Balaji M, Koschorke M, et al. Effectiveness of a communitybased intervention for people with schizophrenia and their caregivers in India (COPSI): A randomised controlled trial. Lancet [Internet]. 2014;383(9926):1385-94. Available from:

http://dx.doi.org/10.1016/S0140-6736(13)62629-X 
10. Agrest M, Le PT, Yang L, Alves-Nishioka S, Alvarado R, Susser ES. Implementing a community-based task-shifting psychosocial intervention for individuals with psychosis in Chile: Perspectives from users. Int J Soc Psychiatry. 2019;65(1):38-45.

11. Chatterjee S, Naik S, John S, Dabholkar H, Balaji M, Koschorke M, et al. Eff ectiveness of a community-based intervention for people with schizophrenia and their caregivers in India ( COPSI ): a randomised controlled trial. Lancet [Internet]. 2014;383(9926):1385-94. Available from:

http://dx.doi.org/10.1016/S0140-6736(13)62629-X

12. Gureje O, Appiah-poku J, Bello T, Kola L, Araya R, Chisholm D, et al. Effect of collaborative care between traditional and faith healers and primary health-care workers on psychosis outcomes in Nigeria and Ghana ( COSIMPO ): a cluster randomised controlled trial. Lancet. 2020;396.

13. Ferrinho P, Omar C. The human resources for health situation in Mozambique. Africa Reg Hum Dev Work Pap Ser No 91. 2006;(91).

14. USAID. Health for the People: National Community Health Worker Programs from Afghanistan to Zimbabwe [Internet]. 2020. 247-256 p. Available from: https://pdf.usaid.gov/pdf_docs/PA00WKKN.pdf

15. Partners A and C. COUNTRY PROFILE: MOZAMBIQUE Mozambique Community Health Programs. 2013.

16. Ndima SD, Sidat M, Give C, Ormel H, Kok MC, Taegtmeyer M. Supervision of community health workers in Mozambique: a qualitative study of factors influencing motivation and programme implementation. Hum Resour Health [Internet]. 2015;1-10. Available from:

http://dx.doi.org/10.1186/s12960-015-0063-x

17. Michie S, Stralen MM Van, West R. The behaviour change wheel: A new method for characterising and designing behaviour change interventions The behaviour change wheel: A new method for characterising and designing behaviour change interventions. Implement Sci [Internet]. 2011;6(1):42. Available from: http://www.implementationscience.com/content/6/1/42

18. Tong A, Sainsbury P, Craig J. Consolidated criteria for reporting qualitative research ( COREQ ): a 32item checklist for interviews and focus groups. Int J Qual Heal Care. 2007;19(6):349-57.

19. Robinson OC. Sampling in Interview-Based Qualitative Research: A Theoretical and Practical Guide. Qual Res Psychol. 2014;11(1):25-41.

20. World Health Organization. mhGAP training manuals for the mhGAP Intervention Guide for mental, neurological and substance use disorders in non-specialized health settings- version 2.0. Vol. 0, World Health Organization. 2017.

21. Braun V, Clarke V. Using thematic analysis in psychology. Qual Res Psychol. 2006;3(2):77-101. 
22. Saldana J. The Coding Manual for Qualitative Researchers. 2009.

23. Hennink MM, Kaiser BN, Weber MB. What Influences Saturation? Estimating Sample Sizes in Focus Group Research. Qual Health Res. 2019;

24. Chilale HK, Silungwe ND, Gondwe S, Masulani-mwale C. Clients and carers perception of mental illness and factors that influence help-seeking: Where they go first and why. Int J Soc Psychiatry. 2017;

25. Wood L, Alsawy S. Recovery in Psychosis from a Service User Perspective: A Systematic Review and Thematic Synthesis of Current Qualitative Evidence. Community Ment Health J [Internet]. 2017;0(0):0. Available from: http://dx.doi.org/10.1007/s10597-017-0185-9

26. Mutiso VN, Musyimi CW, Nayak SS, Musau AM, Rebello T, Nandoya E, et al. Stigma-related mental health knowledge and attitudes among primary health workers and community health volunteers in rural Kenya. Int J Soc Psychiatry. 2017;

27. Mckibbin CL, Kitchen KA, Wykes TL, Lee AA. Barriers and Facilitators of a Healthy Lifestyle Among Persons with Serious and Persistent Mental Illness: Perspectives of Community Mental Health Providers. Community Ment Health J. 2014;566-76.

28. Ross LE, Vigod S, Wishart J, Waese M, Spence JD, Oliver J, et al. Barriers and facilitators to primary care for people with mental health and / or substance use issues: a qualitative study. BMC Fam Pract. 2015;1-13.

29. Asher L, Hanlon C, Birhane R, Habtamu A, Eaton J, Weiss HA, et al. Community-based rehabilitation intervention for people with schizophrenia in Ethiopia (RISE ): a 12 month mixed methods pilot study. BMC Psychiatry. 2018;1-17.

\section{Supplementary Files}

This is a list of supplementary files associated with this preprint. Click to download.

- Supplementarymaterials.docx 